\title{
A CO-FISH assay to assess sister chromatid segregation patterns in mitosis of mouse embryonic stem cells
}

\author{
Stephan Sauer • Sandra S. Burkett • \\ Mark Lewandoski • Amar J. S. Klar
}

(C) Springer Science+Business Media Dordrecht (Outside the USA) 2013

\begin{abstract}
Sister chromatids contain identical DNA sequence but are chiral with respect to both their helical handedness and their replication history. Emerging evidence from various model organisms suggests that certain stem cells segregate sister chromatids nonrandomly to either maintain genome integrity or to bias cellular differentiation in asymmetric cell divisions. Conventional methods for tracing of old
\end{abstract}

Responsible Editors: Helder Maiato and Yves Barral.

S. Sauer · A. J. S. Klar

Gene Regulation and Chromosome Biology Laboratory,

Frederick National Laboratory for Cancer Research,

National Institutes of Health,

Frederick, MD 21702, USA

\section{S. S. Burkett}

Molecular Cytogenetics Section, Frederick National

Laboratory for Cancer Research,

National Institutes of Health,

Frederick, MD 21702, USA

S. Sauer $\cdot$ M. Lewandoski

Cancer and Developmental Biology Laboratory, Frederick

National Laboratory for Cancer Research, National

Institutes of Health,

Frederick, MD 21702, USA

A. J. S. Klar $(\bowtie)$

Gene Regulation and Chromosome Biology Laboratory,

National Cancer Institute, Center for Cancer Research,

National Institutes of Health,

Building 539, Room 154,

Frederick, MD 21702-1201, USA

e-mail: klara@mail.nih.gov vs. newly synthesized DNA strands generally lack resolution for individual chromosomes and employ halogenated thymidine analogs with profound cytotoxic effects on rapidly dividing cells. Here, we present a modified chromosome orientation fluorescence in situ hybridization (CO-FISH) assay, where identification of individual chromosomes and their replication history is achieved in subsequent hybridization steps with chromosome-specific DNA probes and PNA telomere probes. Importantly, we tackle the issue of BrdU cytotoxicity and show that our method is compatible with normal mouse ES cell biology, unlike a recently published related protocol. Results from our CO-FISH assay show that mitotic segregation of mouse chromosome 7 is random in ES cells, which contrasts previously published results from our laboratory and settles a controversy. Our straightforward protocol represents a useful resource for future studies on chromatid segregation patterns of in vitro-cultured cells from distinct model organisms.

Keywords Asymmetric cell division mechanism. somatic strand segregation - mouse stem cell biology and cell differentiation

$\begin{array}{ll}\begin{array}{l}\text { Abbreviations } \\ \text { CO-FISH }\end{array} & \begin{array}{l}\text { Chromosome orientation fluores- } \\ \text { cence in situ hybridization }\end{array} \\ \text { DNA } & \begin{array}{l}\text { Deoxyribonucleic acid } \\ \text { mat1 }\end{array} \\ \text { S-phase } & \text { MNing-type gene 1 } \\ \end{array}$




BrdU
dCTP
$\mu \mathrm{M}$
chr. 7
SCE
WW:CC
segregation

segregation

WC:WC segregation

SSIS

wt

ESC

loxP

Cre

Hprt

$\mathrm{X}$ segregation

Z segregation

\section{UV}

LASER

PMT

Calcein AM

SSEA-1

LIF

$2 \mathrm{i}+\mathrm{LIF}$

\section{AP}

5-bromo-2'-deoxyuridine

Deoxycytidine triphosphate

Micromolar

Chromosome 7

Sister chromatid exchange

Describes the segregation of sister

chromatids of equal replication

history of one pair of homologous

chromosomes to the same sister cells

in mitosis. Old "Watson"-strand-

containing sister chromatids segre-

gate together and old "Crick"-strand-

containing sister chromatids segre-

gate together

Describes the segregation of sister

chromatids of opposite replication

history of one pair of homologous

chromosomes to the same sister cells

in mitosis. One old "Watson"-strand-

containing sister chromatid and one

old "Crick"-strand-containing sister

chromatid segregate together

Strand-specific imprinting and selec-

tive segregation hypothesis for asym-

metric cell division

Wild type

Embryonic stem cell

Locus of crossing over P1

Causes recombination gene

Hypoxanthine-guanine

phosphoribosyltransferase

Recombined products of a mitotic recombination event in G2 segregate away from each other (into different sister cells)

Recombined products of a mitotic recombination event in G2 segregate together (into the same sister cell)

\section{Ultraviolet}

light amplification by stimulated emmission of radiation

Photomultiplier tube

Acetomethoxy derivate of calcein

Stage-specific embryonic antigen 1

Leukemia inhibitory factor

Serum-free medium for ESC cultivation containing a combination of two small molecule inhibitors (2i) and LIF Alkaline phosphatase

\section{Introduction}

DNA replication produces sister chromatids that are chiral with respect to their replication history because alternating leading- and lagging-strand-replicated areas emerge in a mirror-image symmetry between sister chromatids. Molecular and genetic evidence from Schizosaccharomyces pombe mating-type switching has demonstrated that asymmetric developmental decisions exploit the asymmetric nature of sister chromatids and DNA replication (Klar 2007). In particular, inheritance of lagging-strand-synthesized mat1 gene confers competence for mating-type switching through strand-specific imprinting via a DNA transposition mechanism. A highly similar mating-type switching mechanism was also recently identified in Schizosaccharomyces japonicus, a yeast that is otherwise evolutionarily vastly unrelated to $S$. pombe (Yu et al. 2013). We have previously proposed that replication-coupled, epigenetic differentiation of sister chromatids may also occur in higher, diploid eukaryotes to guide asymmetric or strictly symmetric cell divisions (Fig. 1a). In the first step of our model, the asymmetric nature of DNA replication in combination with chromatin assembly would provide opportunities to install strand-specific epigenetic imprints on sister chromatids. Next, segregation of epigenetically differentiated sister chromatids is hypothesized to be coordinated in mitosis, such that both old "Watson" (W)-template-strand-containing sister chromatids are segregated to one sister cell, and both old "Crick" (C)-template-strand-containing sister chromatids are segregated to the other sister cell (WW:CC segregation, shown in Fig. 1a). We have termed the model SSIS, for strand-specific imprinting and selective segregation (Klar 1994).

Experimental support for our model has recently been provided by work carried out in the Horvitz and Stillman laboratories (Nakano et al. 2011). The authors have shown that bilateral neuronal asymmetry in Caenorhabditis elegans brain is dependent on locus-specific priming by asymmetric, lagging- vs. leading-strand chromatin assembly in the great-greatgrandmother cell. Coupled to selective sister chromatid segregation, epigenetically differentiated sister chromatids were proposed to be selectively segregated to sister cells, causing developmental asymmetry. Likewise, we have recently pointed out that the phenotype of certain C. elegans olfactory neuronal asymmetry mutant 
A

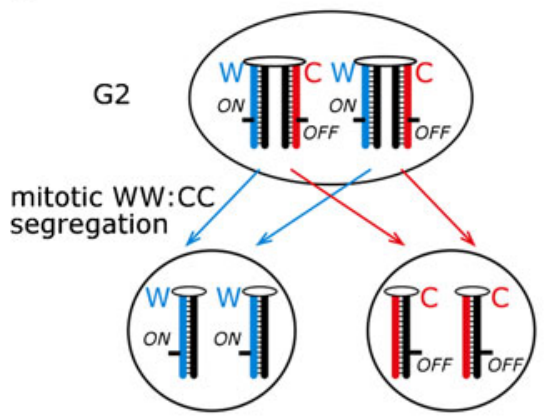

B

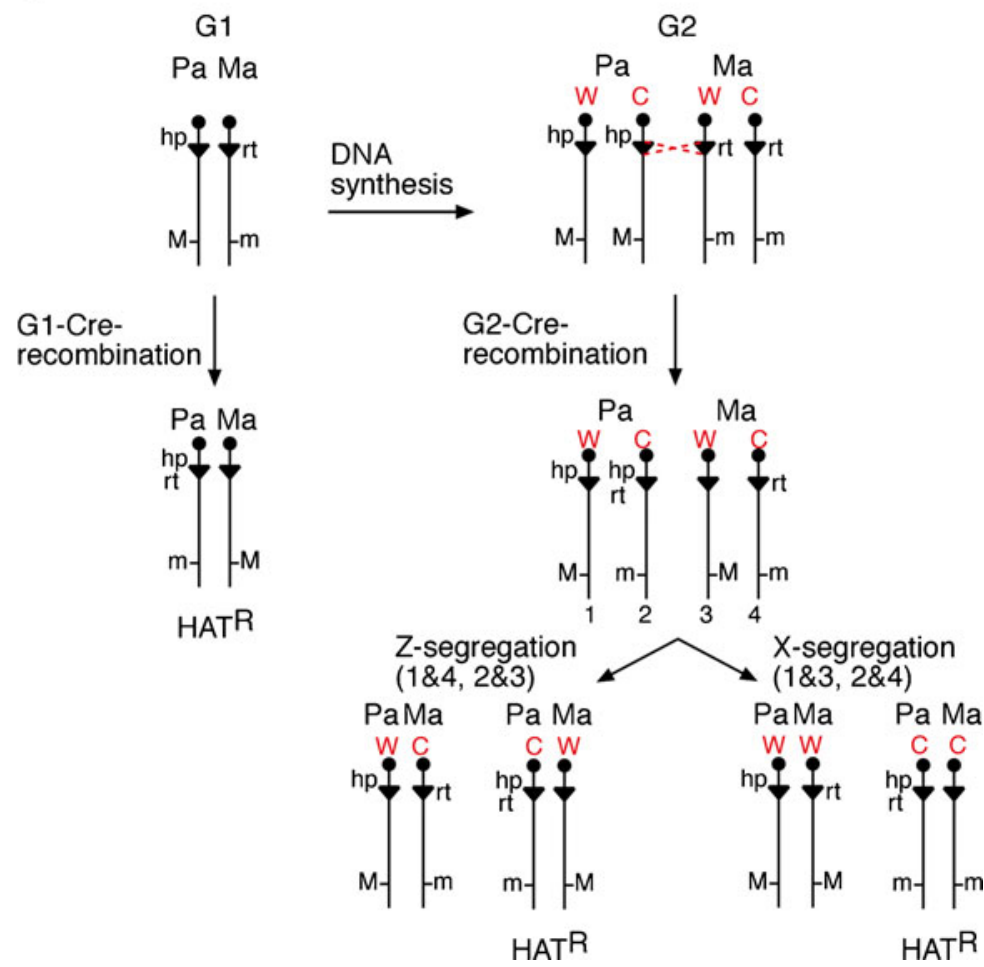

Fig. 1 SSIS hypothesis and Liu et al. (2002) experiment. a The SSIS hypothesis predicts that asymmetrically dividing stem cells epigenetically differentiate sister chromatids during Sphase. This is followed by asymmetric sister chromatid segregation to daughter cells. Here, older W-template-strand containing sister chromatids are segregated to one sister cell, and older C-template-strand containing sister chromatids are segregated to the other sister cell, to constitute a DNA-based mechanism of asymmetric cell division. b Outline of Liu et al.'s (2002) experiment (black). 5' ("hp") and 3' ("rt") parts of the selectable hprt minigene, flanked by a single loxP site (triangle) are placed in identical genomic positions on homologous chromosomes (line with filled circle on top as centromere). Centromere distal to the loxP site is a heterozygous marker ( $M$ on the "paternal" homolog and $m$ on the "maternal" homolog). Cre

supports selective chromatid segregation in the asymmetrically dividing mother cell (Sauer and Klar 2012).

It remains however technically challenging to molecularly test for strand-specific imprinting and biased chromatid segregation due to two main reasons: neither do we know the nature of the proposed epigenetic imprint nor are protocols available that allow for strand segregation studies of individual chromosomes within animal tissues. Hence, only genetic evidence supporting our SSIS model is currently available for diploid organisms (Sauer and Klar 2012). "Indirect" evidence for a SSIS mechanism operating in mouse recombination restores the hprt minigene and confers HAT resistance $\left(H A T^{R}\right)$. Please note that after recombination, only centromere-proximal areas maintain their original $\mathrm{Ma}$ and $\mathrm{Pa}$ identities. When Cre recombination happens in G2, recombined products can either segregate together ( $Z$ segregation) or away (X segregation) from each other. $\mathrm{Z}$ and $\mathrm{G} 1$ recombination do not change the original heterozygous $(\mathrm{M} / \mathrm{m})$ marker. X segregation leads to homozygosis $\left(m / m\right.$ HAT $^{\mathrm{R}}$ cells). DT1E9 ESCs, where the hprt-loxP cassettes were placed centromere-proximal on chr. 7 always undergo $X$ segregation. An explanation for this was previously provided by us and indicated in red. During G2 recombination, only old $\mathrm{W}$-strand- and old C-strand-containing sister chromatids are allowed to recombine. This is followed by selective WW:CC segregation in mitosis, giving rise to homozygosed $(\mathrm{m} / \mathrm{m}) \mathrm{HAT}^{\mathrm{R}}$ cells only

embryonic stem cells (ESCs) has been provided by studies from the Jenkins/Copeland and our laboratories (Liu et al. 2002; Armakolas and Klar 2006, 2007). Liu et al. had engineered transgenic ESCs harboring reciprocal parts of a HPRT minigene coupled to a loxP site, in identical genomic locations on homologous chromosomes. As illustrated in Fig. 1b, Cre-mediated interchromosomal recombination restores the HPRT minigene, and after HAT selelction colonies can be analysed for an initially heterozygous distal marker $(m / M)$ to assess whether recombined chromatids had segregated together (called $\mathrm{Z}$ segregation, maintaining 
heterozygous constitution for marker centromeredistal to the crossover point) or away from each other (X segregation, homozygosis of marker) in mitosis. It is important to note that in this setup, G1 recombination is indistinguishable from $\mathrm{Z}$ segregation (Fig. 1b). Remarkably, exclusive X segregation was observed in an ESC line (DT1E9) where transgenic integration sites were placed centromere-proximal on chromosome (chr.) 7. We proposed previously (Armakolas and Klar 2006) that this bias in mitotic Cre/loxPmediated recombination could represent a case of selective chromatid recombination followed by selective sister chromatid segregation (critical steps of our hypothesis are shown in red in Fig. 1b). In particular, we proposed that only strands of opposite replication history were allowed to recombine (only chromatids containing the old $\mathrm{W}$ strand recombine with chromatids containing the old $\mathrm{C}$ strand), probably due to structural qualities related to opposite helical handedness (Boy de la Tour and Laemmli 1988). If chr. 7 sister chromatids are subjected to "asymmetric" WW:CC segregation in ESCs, then only X segregants would develop (Fig. 2b, lower panel). Interestingly, we also found that the segregation pattern changed upon cellular differentiation. DT1E9-derived endoderm cells maintained exclusive $\mathrm{X}$ segregation, neuroectoderm showed exclusive $\mathrm{Z}$ segregation and three other cell types showed a random mix of $X$ and $\mathrm{Z}$ segregants. The finding that left-right dynein (lrd) mRNA expression was evident in ES, endoderm and neuroectoderm cells but not in cell types that showed random ( $\mathrm{X}$ and $\mathrm{Z}$ ) segregation, further supported the case for chr. 7 being subjected to selective segregation in mitosis (Armakolas and Klar 2007). RNAimediated knock-down of $l r d$ led to emergence of a mix of $\mathrm{X}$ and $\mathrm{Z}$ segregants after Cre/loxP-mediated mitotic recombination. $L r d$ was chosen as a candidate gene because the phenotype of two different null alleles supports its involvement in selective chromatid segregation during a hypothetical left-right axis-generating asymmetric cell division (Sauer and Klar 2012).

Here, we present a cytogenetic assay, based on the chromosome orientation fluorescence in situ hybridization (CO-FISH) method that allows for assessment of sister chromatid segregation patterns of individual chromosomes of in vitro cultured cells. We detect random segregation of mouse chr. 7 and 11 in wildtype (wt) mouse ESCs. Our results are in agreement with a recently published protocol (Falconer et al.
2012); however, we provide evidence that this method is highly toxic to ESCs and arguably to other rapidly dividing cell types also. Because our protocol determines a sister chromatid's replication history or "strandedness" information using telomere repeat probes, it is easily adaptable to various vertebrate cell types amenable for in vitro cultivation.

\section{Results}

Developing a CO-FISH assay to study chromatid segregation in mitosis

To study segregation patterns of sister chromatids in mitosis directly, we devised a modified CO-FISH approach (Fig. 2a). A detailed description of the protocol can be found in the materials and methods. We first assessed lengths of cell cycle stages in a time course experiment, by BrdU pulse-chase and DNA content analysis. This revealed that wt JM8.N4 ESCs have a doubling time of about $14.5 \mathrm{~h}$, and complete Sphase in about $6 \mathrm{~h}$ (data not shown). We reasoned that BrdU exposure should be slightly longer than one Sphase, and cultured ESCs in BrdU-containing medium for $7 \mathrm{~h}$. Next, BrdU is washed out and cells are cultured for the length of one cell cycle in standard growth medium (without BrdU), with subsequent mitotic arrest by addition of colchicine. Metaphase chromosomes are then prepared and the BrdU-containing strand is degraded using a combination of Hoechst 33258 staining, UV irradiation and exonuclease III treatment. Chromosomes are then hybridized overnight using a biotin- or fluorescence-labeled chromosome-specific FISH probe, and after standard washes, PNA probes are hybridized to the telomeres (sense and anti-sense, consecutively) to acquire strand information.

As shown in Fig. 2a, this protocol allows to discriminate between chromatid segregation patterns of the WW:CC and WC:WC type. In WC:WC chromatid segregation pattern, sister chromatids containing one old W-template strand and one old C-template strand segregate together to both sister cells (Fig. 2a, top). The other class comprises cells that segregated sister chromatids of the same replication history together to each sister cell, the so called WW:CC pattern (Fig. 1b, bottom). Hybridization with differentially labeled probes for the sense and anti-sense telomere repeat 


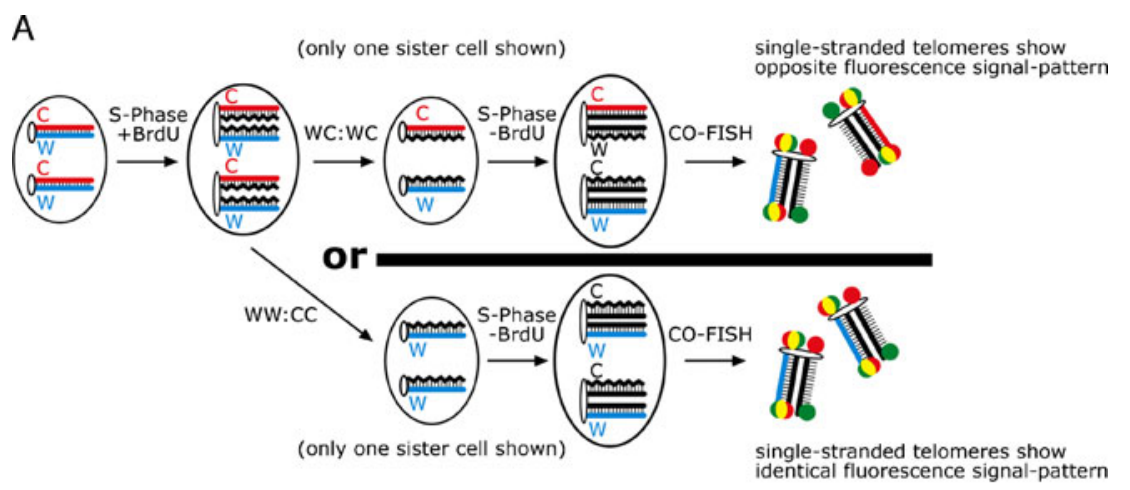

B
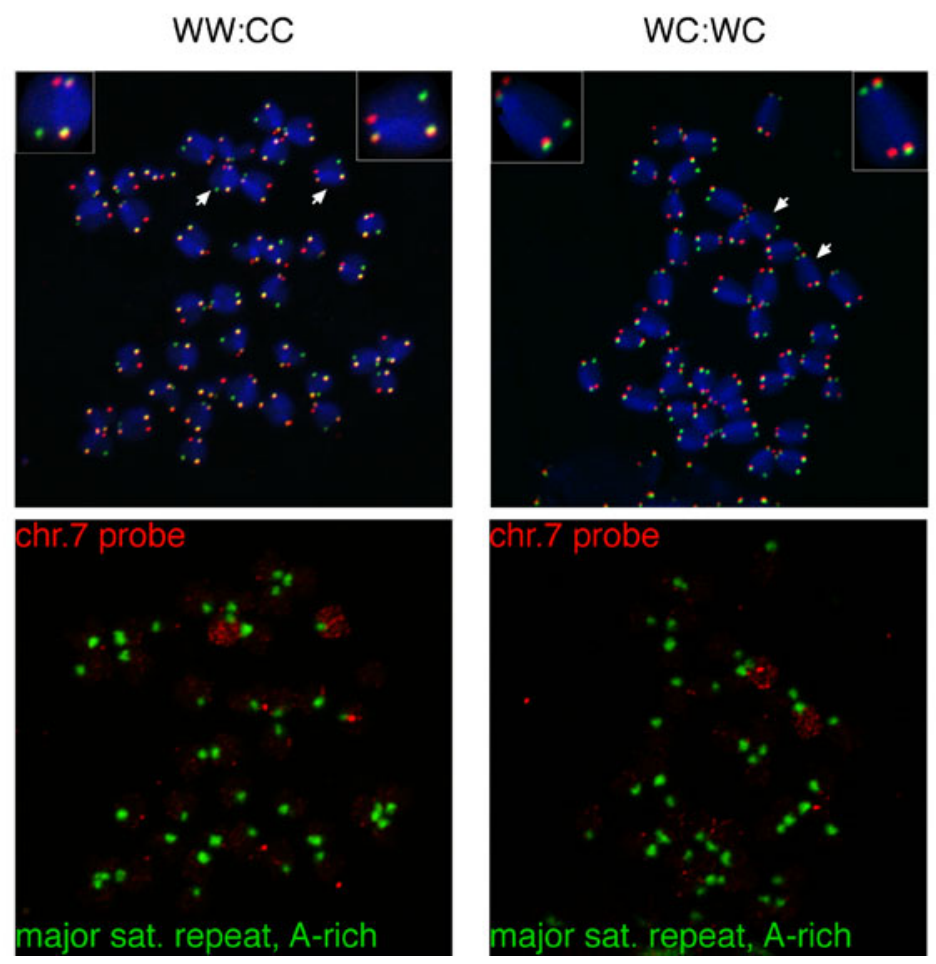

Fig. 2 Our modified CO-FISH assay. a Schematic overview of our CO-FISH assay. A diploid cell with one pair of homologous chromosomes, containing Watson $(W ;$ blue $)$ and Crick $(C$; red $)$ strands is shown. BrdU present in the culture medium is incorporated into growing DNA chains during S-phase. The cell in G2 now contains four sister chromatids, each containing one strand of newly synthesized, BrdU-substituted DNA (indicated by zig-zag-shaped line). Two possibilities for sister chromatid segregation exist: (1) The WC:WC pattern is shown on top, where one old $\mathrm{W}$ - and one old C-template-strand-containing sister chromatid are segregated together to sister cells. (2) In the WW:CC pattern (bottom), sister chromatids of the same replication history are segregated together. One sister cell will contain both old W-template-strand-containing sister chromatids, and the other sister cell will contain both older Ctemplate-strand sister chromatids. To simplify presentation, only one sister cell is shown for each segregation pattern. The next cell cycle is advanced in medium without BrdU, and cells are

arrested in metaphase by colchicine treatment. After chromosome preparation and in situ strand degradation, directly labeled PNA probes complementary to sense (blue) and anti-sense (red) telomere repeat are hybridized to chromosomes. Different fluorescent signals from the single-stranded sister chromatid correspond to WC:WC segregation; whereas, identical fluorescent signals from the single-stranded sister chromatid correspond to WW:CC segregation. Because PNA probes invade doublestranded (ds) DNA, the ds telomere will show double fluorescent signals. Individual chromosomes are identified by additional hybridization of a chromosome-specific DNA probe. b Examples of metaphase spreads showing a WW:CC segregation pattern for chromsome 7 on the left, and a WC:WC segregation pattern on the right. DNA was stained with DAPI (blue), sense- and anti-sense telomere probes are shown in green and red. In situ identification of chr. 7 and major satellite repeat is shown in the bottom. White arrows help identify chromsome 7 in the top, and high-resolution images are placed in the top corners 
will result in the single-stranded sister chromatids of a pair of homologous chromosomes exhibiting either different (WC:WC) or the same (WW:CC) fluorescent color signal. Due to PNA probes' ability to invade double-stranded DNA, the double-stranded telomere on each chromatid will display overlapping fluorescence signals. Hence a pair of sister chromatids is expected to display two double-colored telomeres, and two single-colored telomeres of different color (Bailey et al. 2001). It is important to appreciate that use of telomere probes only allows for $\mathrm{W}$ vs. C strand designation with respect to each other. As illustrated in Fig. 2a, telomeres are sufficient to discriminate between the two possible modes of strand segregation (WW:CC vs. WC:WC). Falconer et al. have previously published (Falconer et al. 2010a) combined use of strand-specific PNA probes for telomeres and the pericentromeric major satellite repeats, and arbitrarily assigned the T-rich strand as "C," and the A-rich strand as "W." Although this additional analysis is not required to address our scientific question, we initially included PNA probes for the A-rich satellite repeat in our assay, in order to readily identify centromeres and not solely rely on the DAPI stain for this purpose (see Fig. 2b).

Typical examples of metaphase spreads are shown in Fig. 2b, the top panel shows telomere G repeat in red, telomere $\mathrm{C}$ repeat in green and DNA (DAPI) in blue. The lower panel shows fluorescence signals from the major satellite repeat (green) and the chr. 7-specific probe (red). On the left, both metaphase chr. 7 homologs show identical telomere fluorescence patterns. The single stranded sister chromatid exhibits green fluorescence on the long arm, and red fluorescence on the short arm, which corresponds to WW:CC segregation in the previous cell division (Fig. 2a, bottom). On the right side, in contrast, a pair of chr. 7 homologs is shown that has undergone WC:WC segregation in the last mitosis. Here, sister chromatids containing one old W-template strand and one old C-template strand segregated together to both sister cells (Fig. 2a, top). Both single-stranded short arm and long arm telomerefluorescence signals are different between homologs.

The readout of this experiment is however not always as clear-cut shown in Fig. 2b. In fact, we frequently observed metaphase chromosomes where strand degradation had not succeeded $100 \%$. It is therefore crucial to set laser powers and PMT detection for telomere probes on the confocal microscope into linear range. Figure 3 shows examples of additional six classes of hybridization signal patterns occurring within homologous metaphase chromosomes. In Fig. 3(a), a chromosome is shown where one "single"-stranded telomere (orange arrow) exhibits double fluorescence signals ("1inc.," for incomplete strand removal at one telomere). Because the telomere at the long arm is single-colored red and green fluorescence of the short arm is arguably vastly stronger than red fluorescence, we assign this metaphase to represent a "WW:CC" segregant with high confidence. In Fig. 3(b), the same is true for the long arm single stranded telomeres on both homologous metaphase chromosomes. We assign WW:CC segregation with high confidence. The following three metaphase spreads (Fig. 3(c-e)) exhibit fluorescence patterns that allow only for reduced confidence in their interpretation. In Fig. 3(c), one telomere shows double fluorescence (orange arrow). In this case, it is helpful to look at other chromosomes within the same metaphase spread. If this double-colored pattern is frequently observed, technical reasons (BrdU exposure sub-optimal as cells were not synchronized) are likely responsible. However, even if technical reasons are to blame, it is impossible to exclude the possiblility that this chromosome has undergone sister chromatid exchange (SCE) during the S-phase when BrdU was present in the medium (frequency around $20 \%$, see below and also Fig. 3(f)). Alternatively, recombination with a nonhomologous chromosome could also account for this doublefluorescence signal. Figure 3(d) shows an example of a combination of incomplete strand removal (top right) and double fluorescence signals (low left), Fig. 3(e) shows two double fluorescence signals. Figure 3(f) shows a classic example of a chromsome that has undergone recombination, as single stranded telomeres are in the same orientation. SCE during the "BrdU+" Sphase will lead to such telomere-fluorescent signals. Because BrdU is known to induce SCE, these metaphase spreads were important in our analysis to estimate the rate of SCE per metaphase. However, alternative recombination scenarios could also account for this telomere fluorescence pattern.

We applied our CO-FISH assay to low-passage and feeder-free mouse JM8.N4 ESCs derived from C57/B6 background (Pettitt et al. 2009). We focused our analysis on chr. 7 and 11. Chr. 11 was included as a "negative control," because Liu et al. had shown that Cre/loxP mediated mitotic recombination of chr. 11 homologs produced the expected random mix of $\mathrm{X}$ 

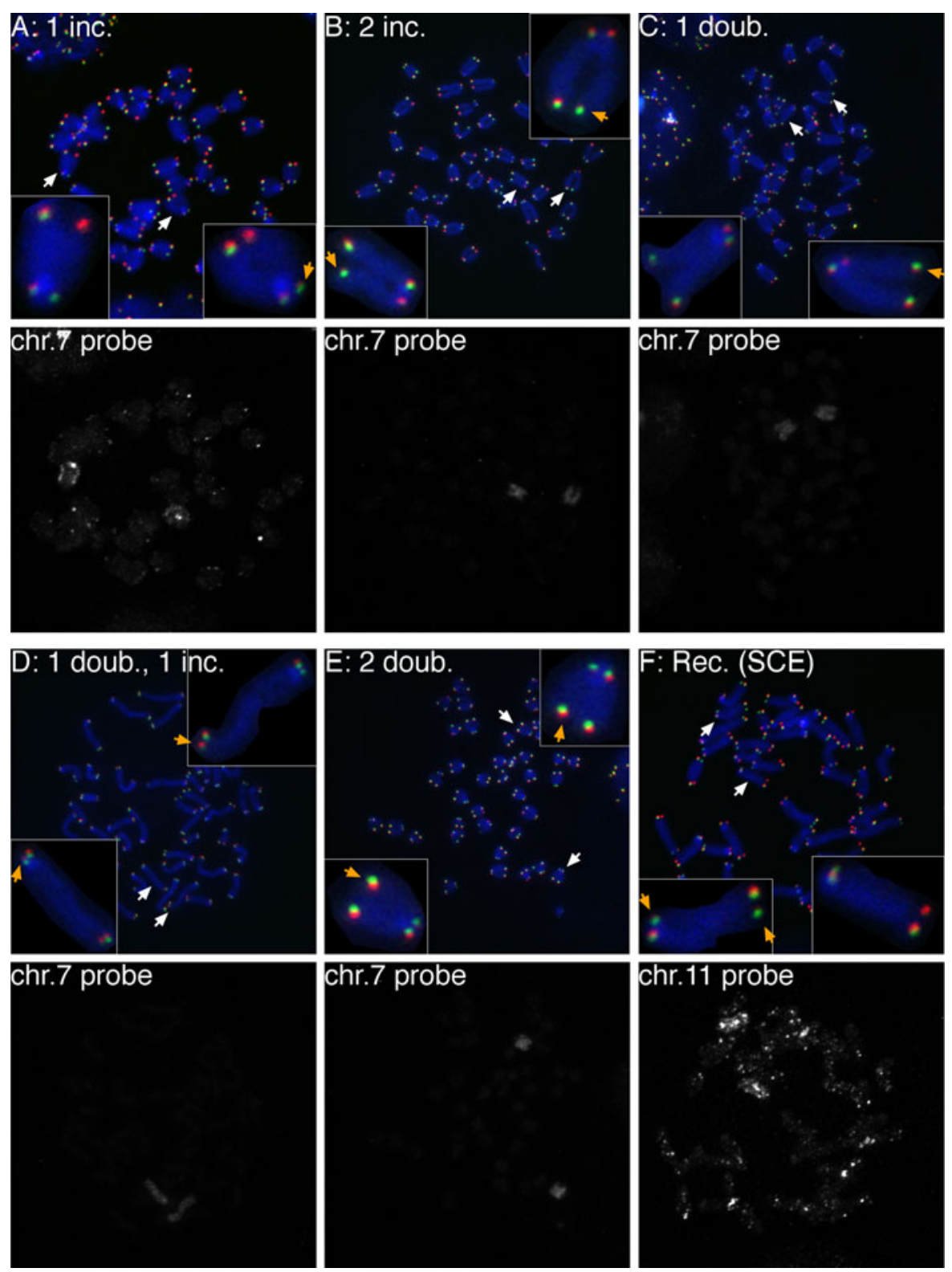

Fig. 3 The other six classes of telomere fluorescence signal patterns. DNA and telomeres are like in Fig. 2, and chr. 7 or 11 is in white on the panel below. $a$ The one telomere incompletely removed class ("1inc."). An orange arrow indicates left-over red fluorescence from an incompletely removed telomere strand. $b$ The two telomeres incompletely removed class ("2inc."). Two orange arrows indicate left-over red fluorescence from an incompletely removed telomere strand on each homolog. Both $(a)$ and $(b)$ are high-confidence WW:CC spreads. $c$ The one telomere shows double fluorescence class ("1doub."). Incomplete strand removal has led to a telomere that shows roughly equal amounts of the two fluorescence signals. This metaphase

and $Z$ segregants (Liu et al. 2002). As summarized in Table 1, analysis of 260 metaphase spreads revealed spread can only be counted as WW:CC with reduced confidence, as recombination cannot be excluded to account for this fluorescence pattern. $d$ The one telomere shows double fluorescence, and one telomere is incompletely removed class (" 1 inc. and 1doub."). A combination of ( $a$ ) and (c), only low confidence in assigning WW:CC pattern. $e$ The two telomeres show double fluorescence class ("2doub."). Like in (c) but on both homologs, only low confidence in assigning WW:CC pattern. $f$ The recombined class of fluorescence pattern ("Rec."). SCE is likely to account for this fluorescence pattern, where single-stranded telomeres display identical single-colour fluorescence. Not useful for assessment of chromatid segregation patterns

random segregation of chr. 7 and 11 sister chromatids in ESCs. We assessed the quality of strand-degradation 


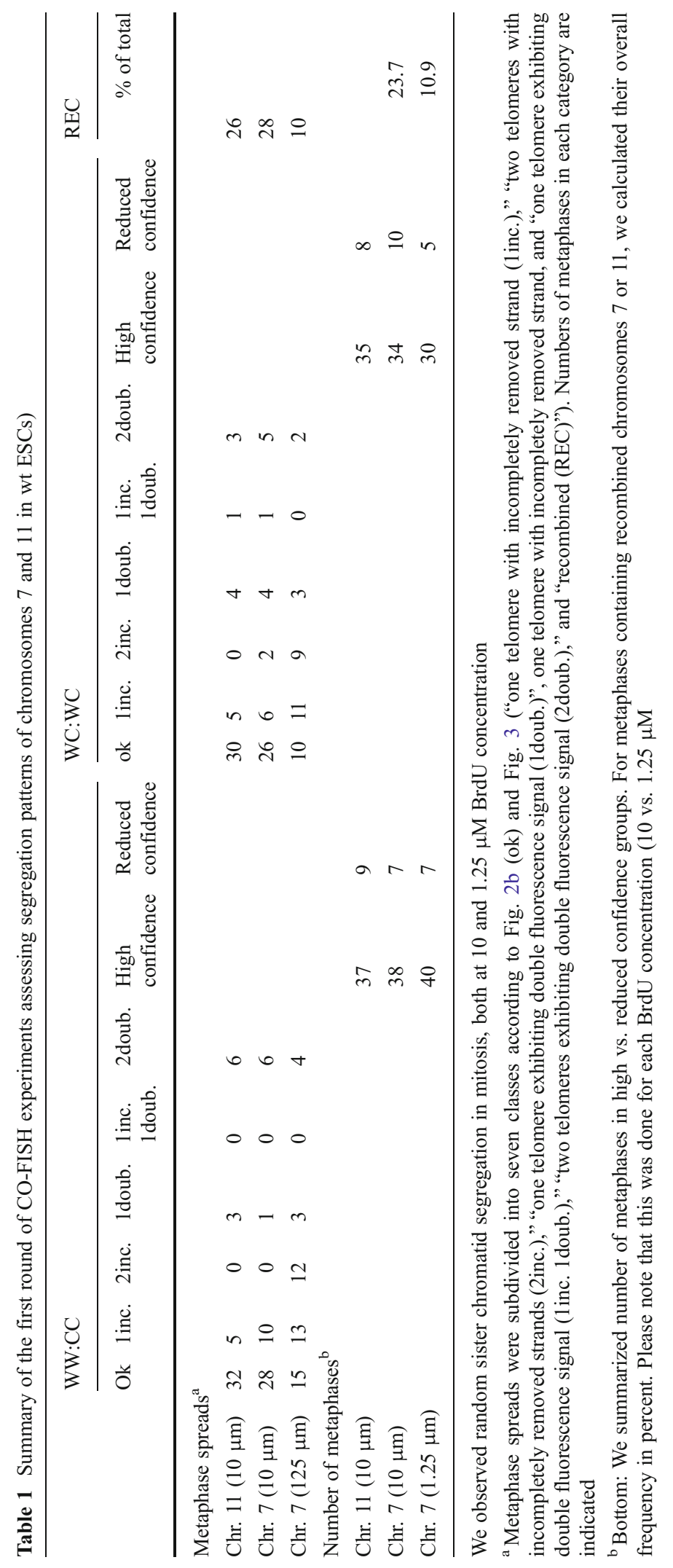


for chr. 7 or 11 in each individual metaphase according to the categories described in Fig. 2b ("ok") and Fig. 3(a-f). The result of this analysis is summarized in Table 1.

When BrdU was used at $10 \mu \mathrm{M}$ concentration, we observed 37 and $38 \mathrm{WW}$ :CC segregants in the highconfidence categories for chr. 7 and 11, respectively. High-confidence WC:WC segregants were seen at almost identical rates (35, chr. 11 and 34, chr. 7). In the reduced-confidence categories, this nine WW:CC vs. eight WC:WC segregants were seen for chr. 11, and seven vs. ten for chr. 7, respectively. Twenty-six (chr. 11) and 28 (chr. 7) metaphase spreads containing "recombined" (as shown in Fig. 3f) chromosomes were also observed. Assuming that all of these represent cases of SCE, then the SCE frequency for a single pair of homologous chromosomes calculates at $23.7 \%$ at $10 \mu \mathrm{M}$ BrdU. Multiplied by 20 chromosomes results in an SCE rate of $\sim 5 /$ metaphase, which is roughly consistent with previous reports (Falconer et al. 2012).

"Recombined" chromosomes are likely a product of SCE, but recombination between homologous or nonhomologous chromosomes is also possible. To better understand the nature of BrdU-induced recombination, we next asked whether BrdU increases recombination between homologous chromosomes in wt ESCs. We assessed the methylation state of the imprinted Snrpn locus on chr. 7 as readout of homologous recombination, according to the procedure of Liu et al. (Liu et al. 2002). Unsynchronized wt ESCs were subjected to BrdU at the same dose and duration as was used in the CO-FISH experiment $(10 \mu \mathrm{M}$ for $7 \mathrm{~h}$ ). Next, cells were trypsinized and plated at low densities for single colony analysis. Homologous recombination, reflected by emergence of di-maternal (methylated) allele-configuration, occurred in 4 out of 60 cases of the $10-\mu \mathrm{M}$ BrdU-treated samples but never in control cells (Fig. 4). This analysis suggests that $10 \mu \mathrm{M}$ BrdU induces mitotic recombination in wt ESCs at rates that exceed recombination rates of the highly recombinogenic $\mathrm{Blm}^{-1-}$ ESCs by two orders of magnitude (Guo et al. 2009). These high levels of recombination were unexpected, and raised the possiblility that randomized chromatid segregation was a result of BrdU-mediated recombination.

We therefore sought to determine whether lowering BrdU concentration can be used for efficient strand degradation. We chose to subject ESCs to the standard CO-FISH procedure, where cells are metaphase

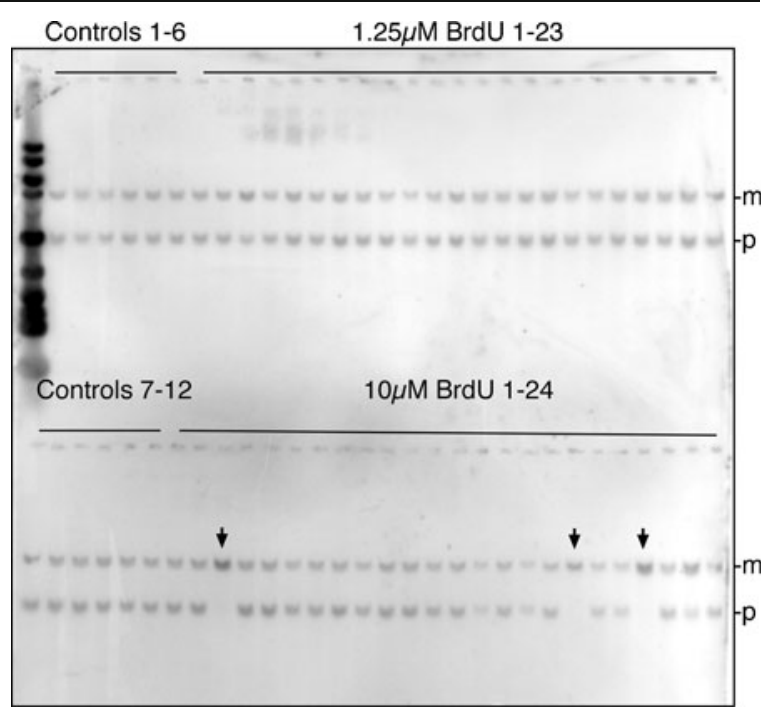

Fig. 4 BrdU induces mitotic recombination between homologous chromosomes in ESCs. Southern blot analysis for methylation imprint at Snrpn promoter, as described in Liu et al. (2002). Taq $\alpha$ I and SacII digest of genomic DNA generates a $\sim 5$-kb band corresponding to the maternal $(\mathrm{m})$ allele and a $\sim 3-\mathrm{kb}$ band corresponding to the paternal $(p)$ epi-allele. Effects of 1.25- and $10-\mu \mathrm{M}$ BrdU exposure for $7 \mathrm{~h}$ were tested on wt JM8.N4 ESCs. In the 10- $\mu$ M sample, three cases of homozygosis to the di-maternal allele configuration were detected on this blot (arrows). In total, we found 4 of 60 homozygosis cases in the $10-\mu \mathrm{M}$ BrdU group, and none in the $1.25-\mu \mathrm{M}$ BrdU or control groups (additional blots not shown)

arrested immediately after 7-h BrdU exposure. We lowered BrdU concentration starting from $10 \mu \mathrm{M}$ in $50 \%$ increments, and tested eight different concentrations (down to one one hundred twenty-eighth of $10 \mu \mathrm{M}$ ). As summarized in Fig. 5, telomere degradation by the CO-FISH method becomes problematic at one sixteenth concentration of $10 \mu \mathrm{M}$, as doublecolored telomeres are starting to occur. Although one sixteenth of $10 \mu \mathrm{M}$ BrdU was compatible with efficient telomere strand degradation in most cases, we reasoned that one eighth $(1.25 \mu \mathrm{M})$ is a better choice, as our CO-FISH protocol requires an additional SPhase in medium without BrdU. We were concerned that during this additional cell cycle, BrdU substitution in DNA might be reduced due to cellular DNA repair mechanisms.

ESCs grown in 1.25 $\mu \mathrm{M}$ BrdU for one S-phase still showed random segregation of chr. 7 sister chromatids in mitosis (40 high confidence WW:CC, 30 highconfidence WC:WC; Table 1, lower panel). The ratio of metaphases exhibiting "recombined" chr. 7 sister chromatids was reduced to $10.9 \%$, consistent with a 

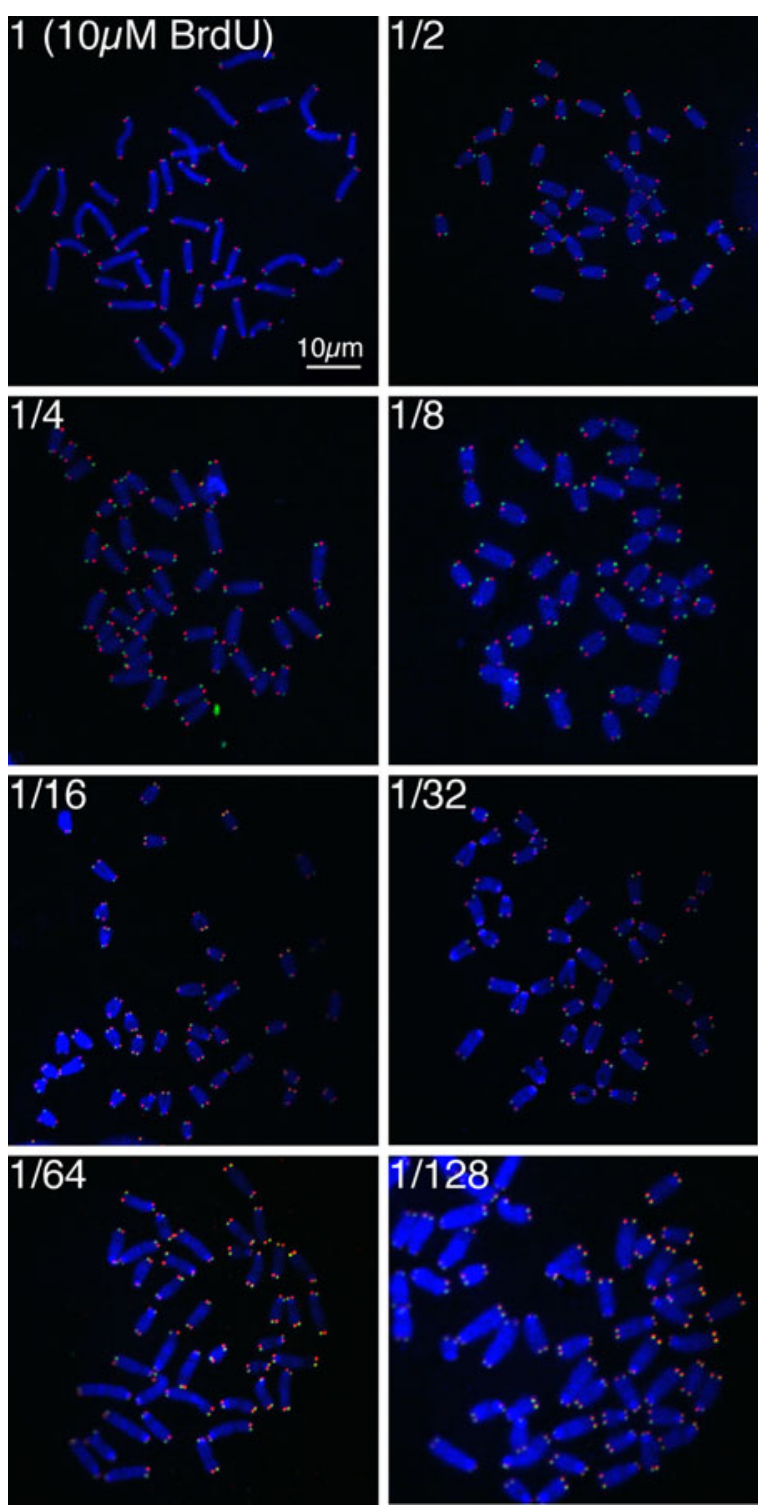

Fig. 5 Effects of lowered BrdU concentrations on telomere fluorescence signals in classic CO-FISH assay. We reduced BrdU concentration in $50 \%$ increments starting from $10 \mu \mathrm{M}$. At one sixteenth concentration, strand degradation becomes inefficient, leading to emergence of double fluorescent signals

dose-dependent mechanism of SCE induction by BrdU. Subsequent Southern blot analysis for chromsome 7 Snrpn methylation imprint showed no case of homozygosis (0/60; Fig. 4).

BrdU exerts profound toxicity on ESCs

We next asked whether growing ESCs in the presence of BrdU would influence biased $\mathrm{X}$ segregation for chr. 7 in
DT1E9 ESCs. Results of three independent experiments are summarized in Table 2. Our first experiment revealed very strong toxicity of $10 \mu \mathrm{M}$ BrdU on ESCs (35 BrdU-treated colonies vs. 1,000 in control sample; $96.5 \%$ lethality), which led us to focus on the effects of lower BrdU concentration. We found significant BrdU toxicity at $1.25 \mu \mathrm{M}$, where $\mathrm{HAT}^{\mathrm{R}}$ colonies emerged at reduced rates (between 40 and $70 \%$ reduction), even under differentiation-suppressing "groundstate" $2 \mathrm{i}+\mathrm{LIF}$ culture conditions (Silva et al. 2008). We speculate that surviving HAT $^{\mathrm{R}}$ colonies are likely to originate from the fraction of ESCs that had spent the least amount of time in S-phase while BrdU was present in the medium, as BrdU exposure was roughly half the length of a cell cycle. Interestingly, $2 \mathrm{i}+\mathrm{LIF}$ fully suppressed $\mathrm{Z}$ segregants, which occurred at $\sim 10 \%$ rate in BrdU-treated samples grown in standard medium. This may indicate that Z segregants represent differentiated ESCs, consistent with a tendency of ESCs to undergo differentiation after exposure to DNA damaging agents (Lin et al. 2005). Notably, we had reported that biased segregation occurs in certain cell types but not in others, thus BrdUinduced differentiation becomes a major concern.

Random chromatid segregation after alleviating BrdU's toxicity

We concluded that BrdU's toxicity on ESCs in colony assays represents a major problem for interpretation of our CO-FISH experiment. This led us to question the nature of BrdU's toxicity. Interestingly, a literature search revealed that BrdU's toxicity correlates with its concentration in the growth medium, rather than with its rate of base-substitution in DNA (Meuth and Green 1974). That is because BrdU-like dTTP-is a potent allosteric inhibitor of ribonucleotide reductase, the rate-limiting enzyme for triphosphorylated deoxyribonucleotide synthesis. BrdU's main detrimental effect on cells is to unbalance the cell's ribonucleotidepool by starving them off deoxycytidine (Ashman and Davidson 1981). Consequently, simultaneous medium substitution with dCTP has been demonstrated to vastly alleviate BrdU's cytotoxic effects, without substantially reducing the rate of BrdU substitution in DNA (Davidson and Kaufman 1978).

We performed colony formation assays on wt JM8.N4 ESCs and tested combinations of several BrdU/dCTP concentrations. We included one very high and long BrdU exposure in these experiments 
Table 2 Summary of three mitotic recombination experiments with DT1E9 ESCs

\begin{tabular}{llll}
\hline & Experiment 1 & Experiment 2 & Experiment 3 (2i+LlF) \\
\hline $10 \mu \mathrm{m}$ BrdU before Cre & 35 (n.d.) & n.d. & n.d. \\
$1.25 \mu \mathrm{m}$ BrdU before Cre & $300(17: 3)$ & $150(18: 0)$ & $370(24: 0)$ \\
$1.25 \mu \mathrm{m}$ BrdU before Cre & n.d. & $150(16: 3)$ & $225(22: 0)$ \\
Control & $1,000(19: 0)$ & $250(19: 0)$ & $740(24: 0)$ \\
\hline
\end{tabular}

Total numbers of $\mathrm{HAT}^{\mathrm{R}}$ colonies are compared. Subsequent $\mathrm{X} / \mathrm{Z}$ segregation ratio determined by Southern blot analysis of the initially heterozygous distal Snrpn marker is shown in brackets. Toxicity of $10-\mu \mathrm{M}$ BrdU in the first experiment had such a profound effect on colony numbers that we did not include it in experiments 2 and 3. Here, only 1.25- $\mu \mathrm{M}$ BrdU concentration was tested and Cre transfection occurred as indicated. We observed low-level emergence ( $\sim 10 \%)$ of $Z$ segregants at $1.25-\mu \mathrm{M}$ BrdU in experiments 1 and 2 but not in experiment 3 where ESCs were cultured in differentiationsuppressing $2 \mathrm{i}+\mathrm{LIF}$ conditions. This may indicate that $\mathrm{Z}$ segregants represent differentiated cells, consistent with ESCs' tendency to differntiate after exposure of DNA damaging agents. Bottom: summary of the experimental workflow of these assays.

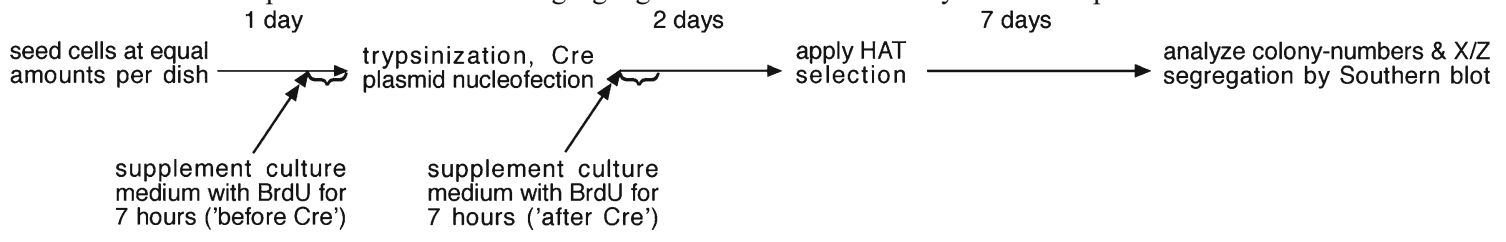

n.d. not done

(40 $\mu \mathrm{M}$ for $16 \mathrm{~h}$ ), because these conditions were used in a recently published study from Peter Lansdorp's laboratory on chromatid segregation in ESCs (Falconer et al. 2012). The authors had performed single cell deep sequencing analysis on BrdU-stranddegraded DNA preparations and found random segregation of all mouse chromosomes in mitosis. The issue of BrdU's cytotoxicity was not addressed in this study.

Because intracellular BrdU can affect ribonucleotide reductase activity after BrdU has been removed from the medium, we kept the dCTP concentration (in samples containing $\mathrm{dCTP}$ ) constant for another $24 \mathrm{~h}$ and reduced it subsequently in $50 \%$ increments each day, for two more days. Colonies were counted 7 days after BrdU removal. As summarized in Fig. 6a, BrdU alone reduced colony numbers in a concentration-dependent fashion. Surviving colonies, as illustrated in Fig. 6b, tested positive for alkaline phosphatase activity, a hallmark of undifferentiated ESCs. At $1.25 \mu \mathrm{M}$, colony numbers dropped to $55 \%$ of control cells, consistent with results obtained in mitotic recombination assays on DT1E9 ESCs. Remarkably, Falconer et al.'s BrdU treatment of $40 \mu \mathrm{M}$ for $16 \mathrm{~h}$ essentially killed off ESCs. We observed an average reduction of colony numbers to $1.5 \%$ of controls, raising concerns about potential artifacts in their results, and adaptability of their method to other cell types. Simultaneous dCTP substitution significantly increased colony formation efficiencies, particularly for the $1.25-\mu \mathrm{M}$ BrdU groups. Here, colony numbers were within the margin of error of that of control cells. Despite slightly higher colony formation efficiencies in 1.25/400 $\mu \mathrm{M}$ (BrdU/dCTP) samples, we chose to go ahead with $1.25 / 200 \mu \mathrm{M} \mathrm{BrdU/dCTP}$ condition (average of $93.6 \%$ colonies of controls), because we found that BrdU-strand degradation in COFISH assay worked more efficiently on $1.25 / 200 \mu \mathrm{M}$ BrdU/dCTP-treated ESCs.

To assess short-term effects of BrdU and dCTP on ESCs, we next performed a live/dead cell viability assay (Invitrogen). Here, we compared control ESCs with samples treated according to our protocol vs. Lansdorp's protocol $(7 \mathrm{~h}, 1.25 \mu \mathrm{M}$ BrdU/200 $\mu \mathrm{M}$ dCTP vs. $16 \mathrm{~h}, 40 \mu \mathrm{M}$ BrdU). ESCs were stained immediately after ( $1 \mathrm{~h}$ time point), and 1 day after end of treatment ( 25 -h time point), and subjected to quantitative (due to addition of counting beads) flow cytometric analysis (Fig. 7a, b). For the second time point, we also included FACS analysis for the ESC surface-marker SSEA-1 in our analysis (Fig. 7c, d). As summarized in Fig. 7a, b, we consistently observed very little effects on live/dead cell ratios immediately after BrdU exposure. However, $24 \mathrm{~h}$ later, effects of $40 \mu \mathrm{M}$ BrdU were evident. Combined BrdU/dCTP treatment had only mild effects on cell proliferation. The live cell/counting bead ratio in relationship to control cells was $79 \%$ for ESCs subjected to our CO-FISH 
A

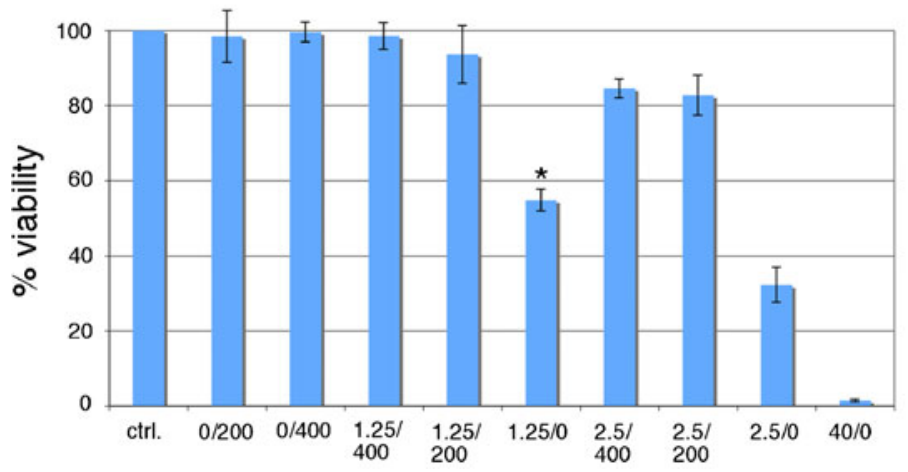

B

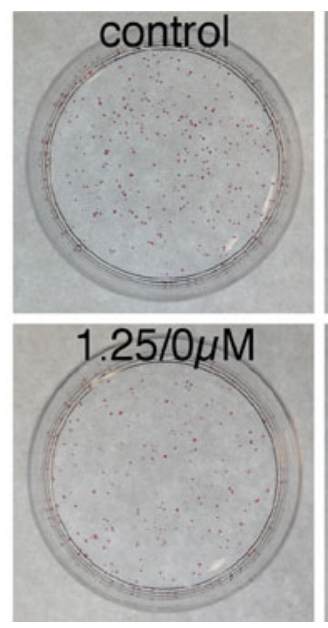

$1.25 / 200 \mu \mathrm{M}$

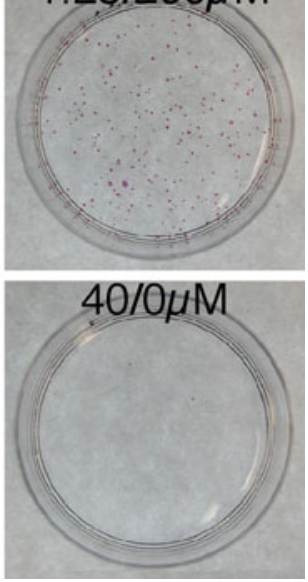

Fig. 6 Effects of BrdU treatment on cell viability. a The bar graph shows colony formation assays of wt JM8.N4 ESCs to test effects of BrdU and BrdU/dCTP combinations at indicated concentrations. BrdU/dCTP concentrations are shown in micromolars. Cells were plated at equal densities on 6-well dishes (day 0), and after 1 day (day 1), were exposed to BrdU, dCTP, or BrdU/dCTP. Treatment time was $7 \mathrm{~h}$, except for $40-\mu \mathrm{M}$ BrdU (16 h). dCTP exposure was kept for another $24 \mathrm{~h}$ and was subsequently reduced in $50 \%$ increments for two more days. On day 2, cells were

BrdU/dCTP conditions; $40 \mu \mathrm{M}$ BrdU, in contrast, caused this rate to decrease to $22 \%$, concomitant with increased occurrence of dead cells (Fig. 7a (bottom), b). SSEA-1 staining (gated on live cells assessed by forward and side scatter) also revealed detrimental effects of $40 \mu \mathrm{M} \mathrm{BrdU}$, as the characteristic peak appears much wider, indicating downregulation of this stem cell marker in a subset of cells (Fig. 7c); $89 \%$ of control cells and $85 \%$ of $1.25 / 200-\mu \mathrm{M}$-treated cells showed high-level expression, compared with $69 \%$ of $40-\mu \mathrm{M}$ BrdU-treated cells (Fig. 7d). In summary, these results were overall consistent with the previously performed colony assays, and showed that ESCs can be subjected to low-level BrdU/dCTP concentrations with limited consequences. The condition used in the Falconer et al. protocol, in contrast, is incompatible with normal ESC biology.

We performed CO-FISH analysis on ESCs subjected to $1.25 / 200 \mu \mathrm{M}$ BrdU/dCTP and studied segregation patterns of chr. 7 and 11 . In addition, we also performed CO-FISH analysis with $2.5 / 400-\mu \mathrm{M}$ BrdU/dCTP-substituted ESCs, but only analyzed 23 metaphase spreads because of the aforementioned problems with strand degradation. As summarized in Table 3, we find random segregation for both chr. 7 and 11: 26 high confidence WW:CC segregants vs. 17 trypsinized and split either 1:65,000 or 1:100,000, and colonies were counted after 1 week. Colony numbers were normalized to untreated control samples. Averages of three experiments are shown including error bars for standard deviations, except for one sample $(1.25 / 0 \mu \mathrm{M}$ BrdU/dCTP, indicated by an asterisk) where the experiments were only carried out twice. b In one experiment, cells were split into 6-cm plates on day 2, and 7 days later colonies were stained for alkaline phosphatase $(A P)$ activity. All colonies were AP positive

high confidence WC:WC segregants for chr. 7 and 19 vs. 12 for chr. 11 , respectively. In the $2.5 / 400-\mu \mathrm{M}$ BrdU/dCTP experiment, we found 9 vs. 6 highconfidence chr. $7 \mathrm{WW}$ :CC vs. WC:WC segregants, recombined chromosomes occurred at a rate of $17.4 \%$. For the $1.25 / 200-\mu \mathrm{M}$ experiment, this group occurred at $16.5 \%$. Both rates are above the $10.9 \%$ seen for $1.25 \mu \mathrm{M}$ alone, which is surprising. These differences can however be explained by the relatively low statistical power of this analysis and the fact that some SCEs might in fact be counted in the reduced confidence groups.

Because we had prepared our chromosome FISH probes with Biotin (chr. 7, detected by Alexa 647) and Texas Red (chr. 11), we combined them for a final experiment to ask whether segregation patterns of chromosomes correlated within individual mitoses. It is in theory possible that chr. 7 and 11 show a random pattern individually, but that their segregation patterns are coordinated (WW:CC with WW:CC and WC:WC with WC:WC). As summarized in Table 4, amongst 15 metaphase spreads analysed, we find that two segregation classes were represented 2 times each, one class was observed 5 times and one class was observed 6 times. We conclude that sister chromatid segregation of chr. 7 and 11 is neither biased nor coordinated in ESCs. 
A

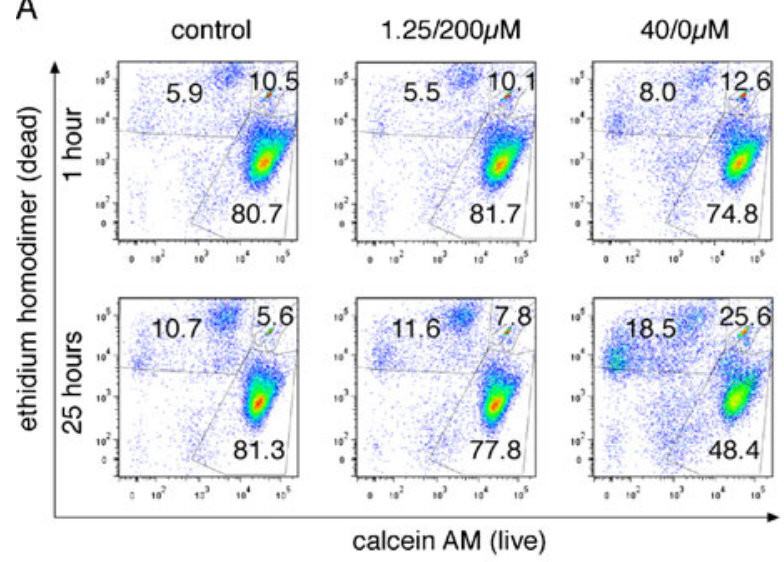

gated populations

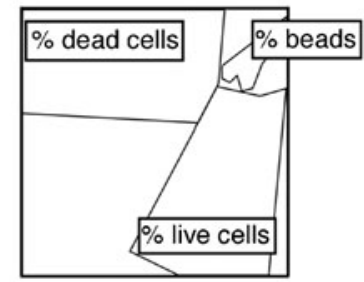

B

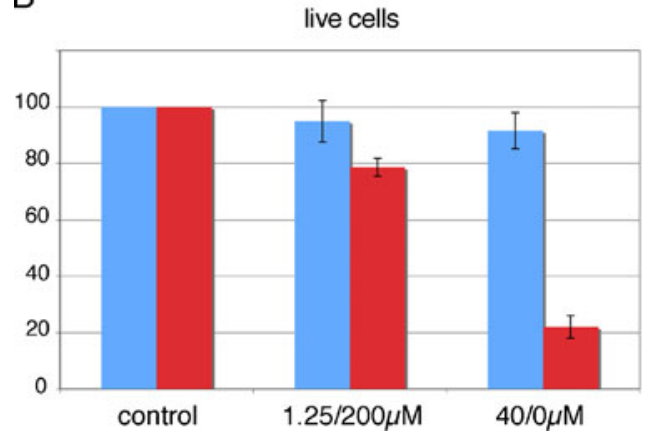

Fig. 7 Comparison of BrdU strand-substitution conditions between the protocol used in this study $(1.25 / 200 \mu \mathrm{M} \mathrm{BrdU} /$ dCTP, $7 \mathrm{~h}$, with $200-\mu \mathrm{M}$ dCTP medium substitution persisting for another day) and the protocol used by Falconer et al. ( $40 \mu \mathrm{M}$ BrdU for $16 \mathrm{~h}$, no dCTP). a FACS-plot compilation of a typical experiment. We gated for live cells (bottom right), dead cells (top left), and counting beads (top corner), which were added as an internal control. Cell preparations were diluted $1: 2$ prior to 25-h analysis. Our analysis revealed subtle differences in live/ dead cell ratios immediately after BrdU treatment (1-h samples). One day later $(25 \mathrm{~h})$, the cytotoxic effects of BrdU, particularly in the $40-\mu \mathrm{M}$ sample, are evident. The fraction of live cells is reduced from $\sim 81$ to $\sim 48 \%$, the number of dead cells is increased by $\sim 70 \%$. The fraction of counting beads has roughly quadrupled ( $\sim 6$ vs. $\sim 25 \%$ ), indicating reduced overall cell numbers. BrdU/dCTP-treated samples,
C

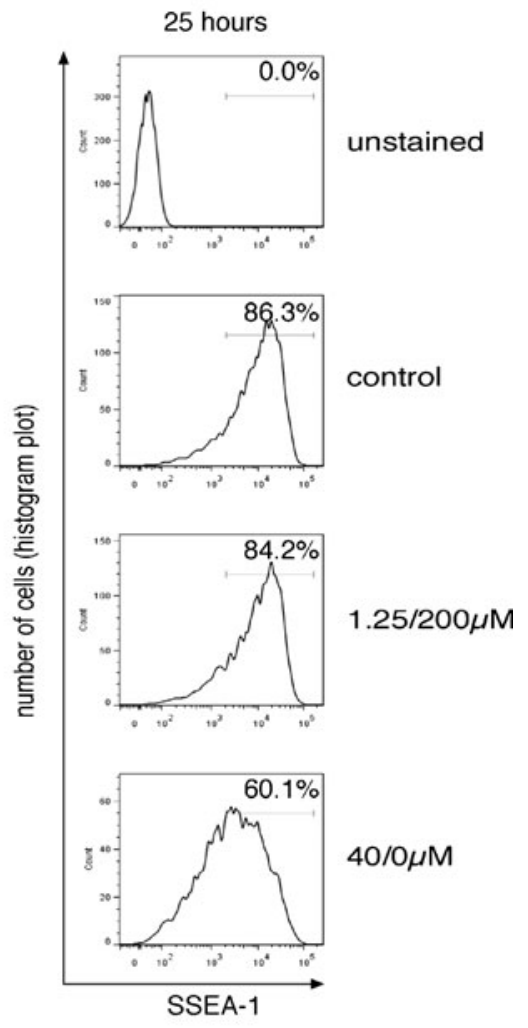

D

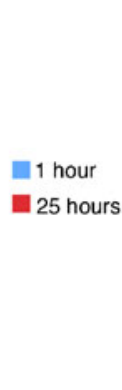

SSEA- $1+$ cells, 25 hours

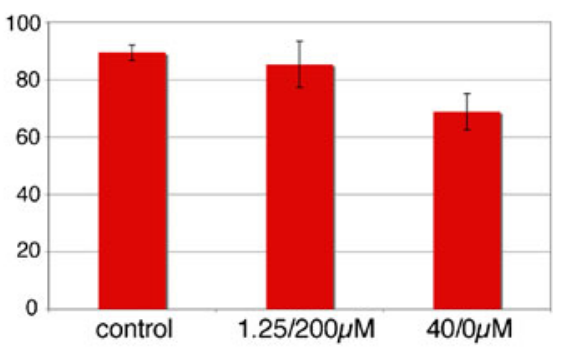

in contrast, are much less affected. b Summary of development of live cell ratios between 1 and $25 \mathrm{~h}$ time-points. Average of three experiments normalized to counting beads and control cells, with error bars showing standard deviations. Whereas $\sim 80 \%$ of control live cells can be detected in the $1.25 / 200-\mu \mathrm{M}$ samples, $40 \mu \mathrm{M}$ BrdU reduces this rate to $\sim 20 \%$. c Histogram plots for stem cell surface marker SSEA-1 expression assessed by flow cytometry, $25 \mathrm{~h}$ after BrdU was removed from the culture medium. We gated on live cells based on forward and side scatter. Whereas control and $1.25 / 200-\mu \mathrm{M}$ samples show highly similar expression profiles, the variation of SSEA-1 expression is much greater in $40-\mu \mathrm{M}$-treated samples, and the mean expression is lowered. d We gated on cells exhibiting high SSEA-1 expression and summarized three staining experiments. Error bars show standard deviations 
Table 4 When both chr. 7 and 11 were analyzed for their sister chromatid's mitotic segregation patterns in mitosis in the same hybridization experiment, no coordination of their segregation patterns was observed

\begin{tabular}{lll}
\hline Chr. 11 & Chr. 7 & \\
\cline { 2 - 3 } & WW:CC & WC:WC \\
\hline WW:CC & 2 & 6 \\
WC:WC & 5 & 2 \\
\hline
\end{tabular}

\section{Discussion}

Asymmetric DNA template strand segregation in mitosis has been postulated to protect stem cells from DNA replication-associated errors ("immortal strand hypothesis"), and to govern asymmetric cell divisions in fission yeast and metazoan development (Klar 2007). Established experimental approaches to study strand-segregation genome-wide usually involve two consecutive rounds of halogenated nucleotide analog labels, and have provided evidence for genome-wide DNA strand co-segregation in certain cell types (Conboy et al. 2007; Booth et al. 2008; Pine et al. 2010; Hari et al. 2011; Rocheteau et al. 2012). Emerging genetic evidence however suggests that also individual chromosomes are subjected to non-random segregation in various laterality-generating cell divisions of C. elegans, mouse and humans (Singh and Klar 2007; Klar 2008; Sauer and Klar 2012). Moreover, studies from our lab have shown that mitotic recombination experiments of mouse ES cell line DT1E9 and at least one of its differentiated progeny showed selective segregation (away from each other) of recombined sister chromatids in G2 (Armakolas and Klar 2006). Biased X segregation was dependent on the left-right axis-determining gene lrd (Armakolas and Klar 2007). Hence, we sought to specifically test our interpretation of biased X segregation, and developed a modified CO-FISH assay that allows to assess sister chromatid segregation of individual chromosomes. We detected random segregation of chr. 7 (and 11) sister chromatids in mitosis and are therefore open for considering alternative explanations for the biased X segregation phenomenon (Liu et al. 2002; Haber 2006; Smith et al. 2007).

Our assay is particularly versatile as it is applicable to any in vitro cultured vertebrate cell line. That is 
because strand information is acquired by telomererepeat hybridization, using commercially available directly labeled PNA probes. We have also demonstrated that BrdU concentrations can be lowered to one eighth of the dose conventionally used in CO-FISH experiments, and that detrimental effects of BrdU are counteracted by addition of dCTP. Hence, we designed our experiment to be well tolerated by ESCs in both short-term FACS-staining experiments, as well as stringent colony formation assays. A recently published alternative method for assessment of sister chromatid segregation patterns, in contrast, did not include any tests on cell viability (Falconer et al. 2012). In this method, BrdU is applied at $32 \times$ higher concentration for more than twice as long as compared with our conditions $(40 \mu \mathrm{M}$ for $16 \mathrm{~h}$ vs. $1.25 \mu \mathrm{M}$ for $7 \mathrm{~h}$ ). Because the Falconer et al. method is based on single cell strand-specific PCR amplification after BrdU-strand degradation, this protocol is arguably more sensitive to incompletely removed BrdU-substituted DNA strands, as they would lead to "noise" in the subsequent deep sequencing reactions. Consequently, it requires higher rate of BrdU substitution of DNA. Because our COFISH assay is read-out by confocal microscopy, incomplete BrdU-strand removal from telomeres is less of an issue. We identified seven distinct classes for qualitative assessment of telomere-hybridization, and applied them to our analysis. When our mild $(1.25 / 200 \mu \mathrm{M}$ $\mathrm{BrdU} / \mathrm{dCTP}$ ) conditions were used, we found a total of 74 metaphases exhibiting high-confidence fluorescence-signal patterns. In contrast, 47 metaphases were of the reduced confidence or "recombined" groups. This $\sim 3: 2$ distribution is acceptable for single chromosome analysis, and analysis for several chromosomes during one hybridization experiment can also be envisioned. A drawback of our approach is however that due to the telomere's small size, SCEs are easily overlooked. Due to limited incidence of SCEs in the Falconer et al. study (average of 8 SCEs per cell), and the fact that we find SCE's at comparable rates, makes us believe that the telomere repeat is suitable to deduct strand information for entire sister chromatids.

We find that toxicity of Falconer et al.'s protocol is evident after $24 \mathrm{~h}$, and manifests in colony formation assays, where less than $2 \%$ of cells survive. It might be argued that since immediate toxicity was comparatively low, the Falconer et al. method might still provide data of biological significance, because ESCs are not required to survive for another week. We would like to point out that ESCs are functionally defined by their ability to contribute to chimera-formation when injected into blastocysts. Given the poor performance in colony formation assays, it is highly unlikely that cells studied in the Falconer et al. paper could meet this functional criterion. Their data should therefore be interpreted with caution. Nevertheless, we confirm their results of random chromatid segregation.

Our assay is designed to work on in vitro cultured cells; however, asymmetrically dividing stem cells are most often found to be dependent on the microenvironment provided by the niche. It is therefore difficult to envision how this assay could be applied to a truly asymmetrically dividing stem cell. We have found one example in the literature where a mouse stem cell line appears to undergo asymmetrical divisions in liquid culture (Ye et al. 2005). The mouse haematopoietic stem cell line EML-1 has been shown to contain two sub-populations of stem cells, based on surface CD34 and Sca-1 expression. Weissman et al. have shown that after lineage depletion, CD34+ and Sca-1+ (double positive (dp)) cells occurred at the same rate as doublenegative $(\mathrm{dn})$ cells. When FACS-sorted dp cells were put in culture, the $1: 1$ ratio of $\mathrm{dp} / \mathrm{dn}$ cells was re-established after approximately four cell divisions. This led the authors to speculate that either a complex signaling feedback mechanism between the two populations exists, or that dp cells are inherently asymmetrically dividing as has been described for cells of $S$. pombe. We suggest that EML-1 cell line is a good material to apply our CO-FISH method in a future study. Moreover, as tissue and organ culture protocols evolve, in vitro studies of various asymmetrically dividing vertebrate cells may sooner or later be technically possible.

\section{Materials and methods}

CO-FISH assay We first assessed cell cycle length of JM8.N4 ESCs by seeding 250,000 cells onto four 6$\mathrm{cm}$ dishes. Because trypsinisation itself might influence cell cycle dynamics, we waited for $17 \mathrm{~h}$ before we counted cells on the first plate ( 0 -h time point). We counted cells of the second, third, and fourth plates after 22.5, 33.5, and $47.75 \mathrm{~h}$, respectively. Counts were $360,000,1.035$ million, 1.970 million, and 
3.401 million. Cell numbers (as fractions of 1,000) were plotted against time in a Microsoft Excel graph, and an exponential trendline applied, which was described as $y=363.74 \mathrm{e}^{0.0478 x}\left(R^{2}=0.9961\right)$. Deriving this formula with respect to $x$, and selecting arbitrary values for $x$ that double, the doubling time calculated as $14.50 \mathrm{~h}$.

We also performed a BrdU pulse-chase experiment (20 $\mu \mathrm{M}$ BrdU for $20 \mathrm{~min}$ ) samples were fixed every $2 \mathrm{~h}$ for $12 \mathrm{~h}$. The protocol we used can be found here: http://www2.iib.uam.es/citometria/pdf/brdu-pi.pdf.

FACS analysis revealed that practically all cells had migrated through S-phase after $6 \mathrm{~h}$; by $8 \mathrm{~h}$, no BrdU containing cell was found in S-phase.

For CO-FISH chromosome preparation, we grew ESCs for $7 \mathrm{~h}$ in presence of BrdU/dCTP $(1.25 / 200 \mu \mathrm{M})$, washed them once and changed to standard medium. After $14.5 \mathrm{~h}$, we added colchicine at $0.25 \mu \mathrm{g} / \mathrm{ml}$ for $3 \mathrm{~h}$. Cells were then hypotonically swollen and fixed as described in (Guo et al. 2005). After the fourth wash in fixative, we kept cells at $-20{ }^{\circ} \mathrm{C}$ overnight. Prior to metaphase spreading cells were washed once in ice-cold fixative.

For metaphase spreads, slides (Corning 2949$75 \mathrm{X} 25$ ) were washed for $5 \mathrm{~min}$ in $100 \%$ ethanolcontaining $1 \%$ concentrated $\mathrm{HCl}$ and air-dried. Metaphase spreads were prepared as described in Henegariu et al. (2001), except that we kept our waterbath temperature between 80 and $85^{\circ} \mathrm{C}$.

For the CO-FISH assay, all enzymatic and staining reactions were performed in a humid chamber and the slide was covered by parafilm. Formamide refers to deionized formamide.

For initial steps we followed a modified protocol describe here (Falconer et al. 2010b), except that pepsin digest was followed by two PBS washes, followed by $0.5 \mathrm{mg} / \mathrm{ml} \mathrm{RNaseA-digest} \mathrm{at} 37{ }^{\circ} \mathrm{C}$, followed by two $2 \times$ SSC washes and Hoechst 33258 staining. We also left out any paraformaldehyde fixation steps. We continued with UV crosslinking in a Stratalinker 2400 for $30 \mathrm{~min}$ at $25{ }^{\circ} \mathrm{C}$ (RT), followed by one wash in PBS and another wash in PBS where the $\mathrm{NaCl}$ concentration was increased to $250 \mathrm{mM}$ (same as NEB buffer 1 used for Exonuclease III treatment). This was followed by $25 \mathrm{~min}$ of Exonuclease III treatment at $37{ }^{\circ} \mathrm{C}$ (NEB M0206L, 1,000 U/slide in $100 \mu \mathrm{l}$ volume), and two washes in $2 \times$ SSC. Next, chromosomal DNA was denatured by transferring slides to $70 \%$ formamide, $30 \% 2 \times \mathrm{SSC}$ for $\sim 100 \mathrm{~s}$ at $72{ }^{\circ} \mathrm{C}$. Slides were then quenched in ice-cold $70 \%$ ethanol and serially dehydrated in 90, 95, and $100 \%$ ethanol at $2 \mathrm{~min}$ each at RT. Slides were subsequently subjected to hybridization with chromosome-specific probes.

Mouse chromosome specific painting probe for chr. 7 and 11 were generated by bivariate chromosome flow sorting followed by degenerate oligonucleotide primed PCR amplification as described (Telenius et al. 1992a; Telenius et al. 1992b) using Tex-Red-12-dUTP (ChromaTide, Invitrogen) and Biotin-16 dUTP (Roche). In situ hybridizations of the mouse probe were performed with about 300-400 ng of each PCR product per probe with $10 \mu \mathrm{g}$ of mouse Cot-1 were precipitated and then dissolved in 10- $\mu$ l hybridization buffer (50\% formamide, $10 \%$ dextran sulfate, and $2 \times$ $\mathrm{SSC})$. The probe and prepared slide were co-denatured at $75{ }^{\circ} \mathrm{C}$ for $5 \mathrm{~min}$ and hybridized in a humidity chamber at $37{ }^{\circ} \mathrm{C}$ overnight. Slides were washed ( 3 min at $72{ }^{\circ} \mathrm{C}$ in $0.4 \times \mathrm{SSC}$ at $0.3 \%$ Triton X-100 followed by two washes at RT in $2 \times \mathrm{SSC}$ at $0.1 \%$ Triton X-100 for 2 min each) and dehydrated (ethanol series) in preparation for telomere probe application.

Telomere probes were prepared and hybridized as described in an online-protocol published by Titia deLange's laboratory: http://delangelab.rockefeller.edu/ protocols_files/CO-FISH.pdf

For direct-labeled chr. 11 probe, we performed a final wash in PBS after the three 5 min washes with hybridization wash buffer No. 2, and mounted slides with DAPI containing Vectashield (H-1200, Vector). For biotin-labeled chr. 7 probe, hybridization wash buffer No. 1 washes were followed by three washes in $4 \times \mathrm{SSC}, 0.05 \%$ Tween-20. Subsequent biotin detection was performed using Streptavidin-Alexa Fluor 647 conjugate (S-32357, Invitrogen) diluted $1: 400$ in $4 \times \mathrm{SSC}, 0.4 \%$ BSA, $0.05 \%$ Tween-20 for 25-30 min at RT. This was followed by two washes in $4 \times$ SSC, $0.05 \%$ Tween-20 and a final wash in PBS and mounting in DAPI-Vectashield.

For microscopic analysis, we used a Zeiss LSM710 confocal microscope with a $\times 63$ oil immersion objective. DAPI, FITC, Cy3, Texas Red, and Alexa 647 were dectected using separate lasers (405, 488, 546, 594, and $633 \mathrm{~nm}$ ), and images were scanned and averaged four times to reduce noise. Final image analysis was carried out using "Zeiss Zen 2009 light" and "Adobe Photoshop CS3" software. 
Cell culture JM8.N4 (Pettitt et al. 2009) and DT1E9 (Liu et al. 2002) ESCs were grown without feeders on gelatinized tissue culture dishes in Knockout DMEM, supplemented with $15 \%$ ES-qualified FCS, $100 \mu \mathrm{M}$ 2-mercaptoethanol, 1× Glutamax, 1× NEAA, 1× penicillin/streptomycin (all from Invitrogen/Gibco), and 1,000 U/ml LIF (Millipore). For $2 \mathrm{i}+\mathrm{LIF}$ conditions, cells were grown on poly-ornithine-treated tissue culture dishes in N2B27 medium (Stem Cell Sciences) supplemented with LIF, $3 \mu \mathrm{M}$ CHIR99021 and $1 \mu \mathrm{M}$ PD0325901 (both from Stemgent). Transient Cre transfection was performed using Lonza's ESC nucleofection kit and a plasmid encoding EF1-alpha driven Cre recombinase (Addgene). Equal numbers of ESCs were compared in each experiment, BrdU treatment occurred either for $7 \mathrm{~h}$ immediately before or after Cre transfection, HAT (Invitrogen) selection started $48 \mathrm{~h}$ after Cre transfection. Snrpnmethylation Southern Blot analysis was carried out as described (Liu et al. 2002). For alkaline phosphatase staining, we used a kit from Stemgent (No. 00-0055); the live/dead viability/cytotoxicity kit was purchased from Invitrogen (L-3224). Both were used according to manufacturers' instructions. FACS analysis was carried out by the Frederick National Laboratory's core facility on a Becton Dickinson Canto II Flow Cytometer and FlowJo analysis software.

Acknowledgments We thank our laboratory colleagues Sharon Moore and Ken Ishikawa for helpful discussions. We would like to thank Dr. Jonathan Keller for sharing his laboratory space and reagents and for helpful comments. Dr. Stephen Lockett, Kim Peifley, and Alla Brafman (Office of the Director) provided outstanding support with Confocal microscope set up and imaging. Kathleen Noer, Guity Mohammadi and Roberta Matthai (Cancer and Inflammation Program) provided help with flow cytometry. The Intramural Research Program of the National Cancer Institute, National Institutes of Health, supports our research. Stephan Sauer is recipient of a long-term fellowship by the International Human Frontier Science Organization (HFSP LT000444/2009).

Conflict of interest The authors declare that they have no conflict of interest.

\section{References}

Armakolas A, Klar AJ (2006) Cell type regulates selective segregation of mouse chromosome 7 DNA strands in mitosis. Science 311:1146-1149
Armakolas A, Klar AJ (2007) Left-right dynein motor implicated in selective chromatid segregation in mouse cells. Science 315:100-101

Ashman CR, Davidson RL (1981) Bromodeoxyuridine mutagenesis in mammalian cells is related to deoxyribonucleotide pool imbalance. Mol Cell Biol 1:254-260

Bailey SM, Cornforth MN, Kurimasa A, Chen DJ, Goodwin EH (2001) Strand-specific postreplicative processing of mammalian telomeres. Science 293:2462-2465

Booth BW, Boulanger CA, Smith GH (2008) Selective segregation of DNA strands persists in long-label-retaining mammary cells during pregnancy. Breast Cancer Res 10:R90

Boy de la Tour E, Laemmli UK (1988) The metaphase scaffold is helically folded: sister chromatids have predominantly opposite helical handedness. Cell 55:937-944

Conboy MJ, Karasov AO, Rando TA (2007) High incidence of non-random template strand segregation and asymmetric fate determination in dividing stem cells and their progeny. PLoS Biol 5:e102

Davidson RL, Kaufman ER (1978) Bromodeoxyuridine mutagenesis in mammalian cells is stimulated by thymidine and suppressed by deoxycytidine. Nature 276:722-723

Falconer E, Chavez E, Henderson A, Lansdorp PM (2010a) Chromosome orientation fluorescence in situ hybridization to study sister chromatid segregation in vivo. Nat Protoc 5:1362-1377

Falconer E, Chavez EA, Henderson A, Poon SS, McKinney S, Brown L, Huntsman DG, Lansdorp PM (2010b) Identification of sister chromatids by DNA template strand sequences. Nature 463:93-97

Falconer E, Hills M, Naumann U, Poon SS, Chavez EA, Sanders AD, Zhao Y, Hirst M, Lansdorp PM (2012) DNA template strand sequencing of single-cells maps genomic rearrangements at high resolution. Nat Methods 9:1107-1112

Guo G, Huang Y, Humphreys P, Wang X, Smith A (2009) A PiggyBac-based recessive screening method to identify pluripotency regulators. PLoS One 6:e18189

Guo J, Jauch A, Heidi HG, Schoell B, Erz D, Schrank M, Janssen JW (2005) Multicolor karyotype analyses of mouse embryonic stem cells. In Vitro Cell Dev Biol Anim 41:278-283

Haber JE (2006) Comment on "Cell type regulates selective segregation of mouse chromosome 7 DNA strands in mitosis". Science 313:1045; author reply 1045

Hari D, Xin HW, Jaiswal K, Wiegand G, Kim BK, Ambe C, Burka D, Koizumi T, Ray S, Garfield S, Thorgeirsson S, Avital I (2011) Isolation of live label-retaining cells and cells undergoing asymmetric cell division via nonrandom chromosomal cosegregation from human cancers. Stem Cells Dev 20:1649-1658

Henegariu O, Heerema NA, Lowe Wright L, Bray-Ward P, Ward DC, Vance GH (2001) Improvements in cytogenetic slide preparation: controlled chromosome spreading, chemical aging and gradual denaturing. Cytometry 43:101-109

Klar AJ (1994) A model for specification of the left-right axis in vertebrates. Trends Genet 10:392-396

Klar AJ (2007) Lessons learned from studies of fission yeast mating-type switching and silencing. Annu Rev Genet 41:213-236 
Klar AJ (2008) Support for the selective chromatid segregation hypothesis advanced for the mechanism of left-right body axis development in mice. Breast Dis 29:47-56

Lin T, Chao C, Saito S, Mazur SJ, Murphy ME, Appella E, Xu Y (2005) p53 induces differentiation of mouse embryonic stem cells by suppressing Nanog expression. Nat Cell Biol 7:165-171

Liu P, Jenkins NA, Copeland NG (2002) Efficient Cre-loxPinduced mitotic recombination in mouse embryonic stem cells. Nat Genet 30:66-72

Meuth M, Green H (1974) Induction of a deoxycytidineless state in cultured mammalian cells by bromodeoxyuridine. Cell 2:109-112

Nakano S, Stillman B, Horvitz HR (2011) Replication-coupled chromatin assembly generates a neuronal bilateral asymmetry in C. elegans. Cell 147:1525-1536

Pettitt SJ, Liang Q, Rairdan XY, Moran JL, Prosser HM, Beier DR, Lloyd KC, Bradley A, Skarnes WC (2009) Agouti C57BL/6N embryonic stem cells for mouse genetic resources. Nat Methods 6:493-495

Pine SR, Ryan BM, Varticovski L, Robles AI, Harris CC (2010) Microenvironmental modulation of asymmetric cell division in human lung cancer cells. Proc Natl Acad Sci U S A 107:2195-2200

Rocheteau P, Gayraud-Morel B, Siegl-Cachedenier I, Blasco MA, Tajbakhsh S (2012) A subpopulation of adult skeletal muscle stem cells retains all template DNA strands after cell division. Cell 148:112-125
Sauer S, Klar AJ (2012) Left-right symmetry breaking in mice by left-right dynein may occur via a biased chromatid segregation mechanism, without directly involving the Nodal gene. Front Oncol 2:166

Silva J, Barrandon O, Nichols J, Kawaguchi J, Theunissen TW, Smith A (2008) Promotion of reprogramming to ground state pluripotency by signal inhibition. PLoS Biol 6:e253

Singh G, Klar AJ (2007) A hypothesis for how chromosome 11 translocations cause psychiatric disorders. Genetics 177:1259-1262

Smith CE, Llorente B, Symington LS (2007) Template switching during break-induced replication. Nature 447:102-105

Telenius H, Carter NP, Bebb CE, Nordenskjold M, Ponder BA, Tunnacliffe A (1992a) Degenerate oligonucleotide-primed PCR: general amplification of target DNA by a single degenerate primer. Genomics 13:718-725

Telenius H, Pelmear AH, Tunnacliffe A, Carter NP, Behmel A, Ferguson-Smith MA, Nordenskjold M, Pfragner R, Ponder BA (1992b) Cytogenetic analysis by chromosome painting using DOP-PCR amplified flow-sorted chromosomes. Genes Chromosomes Cancer 4:257-263

Ye ZJ, Kluger Y, Lian Z, Weissman SM (2005) Two types of precursor cells in a multipotential hematopoietic cell line. Proc Natl Acad Sci U S A 102:18461-18466

Yu C, Bonaduce MJ, Klar AJ (2013) Defining the epigenetic mechanism of asymmetric cell division of Schizosaccharomyces japonicus yeast. Genetics 193:85-94 